\title{
Nuclear imaging of cardiac amyloidosis
}

\author{
Efstathia Andrikopoulou, MD, a and Pradeep Bhambhvani, $\mathrm{MD}^{\mathrm{b}}$ \\ a Division of Cardiovascular Disease, Department of Medicine, The University of Alabama at \\ Birmingham, Birmingham, AL \\ b Division of Molecular Imaging and Therapeutics, Department of Radiology, The University of \\ Alabama at Birmingham, Birmingham, AL
}

Received Jun 16, 2017; accepted Jun 16, 2017

doi: $10.1007 /$ s $12350-017-1028-3$

\section{See related article, pp, 497-504}

Amyloidosis is a multi-system disorder affecting various organs and histologically defined by deposition of abnormal proteins (amyloid fibrils) with characteristic staining pattern. Cardiac involvement may be seen with AL amyloidosis, characterized by deposition of light chains, and TTR amyloidosis, characterized by deposition of transthyretin, either genetically abnormal (mutant transthyretin, familial form of cardiac amyloidosis) or normal (wild-type transthyretin-wt, senile systemic amyloidosis). Cardiac amyloidosis (CA) is an underdiagnosed clinical entity affecting $50 \%$ of the patients with AL amyloidosis, in almost all patients with wt-TTR amyloidosis and with variable frequency of cardiac involvement in mutant-TTR amyloidosis depending on the underlying mutation. ${ }^{1,2}$ Under-diagnosis is partly due to the inherent difficulty in making a definitive diagnosis of CA, for which endomyocardial biopsy remains the gold standard. Tissue-based diagnosis, however, is an invasive approach and it is not uncommon for patients either to be considered too high of a risk to undergo biopsy, or refuse biopsy. In these cases, diagnosis of CA relies on (a) obtaining tissue from a different site (abdominal fat pad, salivary glands etc.) and (b) identifying signs indicative of CA on cardiac magnetic resonance imaging. Another reason why diagnosis of CA is challenging is because its cardinal

Reprint requests: Efstathia Andrikopoulou, MD, Division of Cardiovascular Disease, Department of Medicine, The University of Alabama at Birmingham, Birmingham, AL; eandrikopoulou@bwh.harvard.edu

J Nucl Cardiol 2019;26:505-8.

$1071-3581 / \$ 34.00$

Copyright (C) 2017 American Society of Nuclear Cardiology. echocardiographic features (increased ventricular wall thickness, impaired diastolic function) are also frequently seen with other common disorders, most notably hypertensive heart disease and other restrictive cardiomyopathies.

Despite the difficulty in identifying patients with the disorder, it is important not only to identify patients at early stages of the disease in order to institute appropriate therapy, but also to differentiate between AL-CA and TTR-CA subtypes. The latter is due to their differing prognosis, AL-CA carrying the worst and wt-TTR generally believed to have the most favorable prognosis in terms of patient survival. ${ }^{3,4}$ Timely intervention and initiation of treatment is also essential. In the past, it was common belief that no effective therapy for CA existed; however, this is no longer the case. Patients with AL-CA may see improvement in their survival of up to 12 years with appropriate chemotherapy. ${ }^{5,6}$ The traditional treatment for TTR-CA is liver transplantation, with novel pharmacological agents under development. ${ }^{7}$ In the face of these advances and prognostic implications of CA, it is essential to make the correct diagnosis using prompt and reliable non-invasive imaging modalities. This will allow not only for differentiation amongst the subtypes of CA (AL versus TTR), but also from other myocardial disorders with similar imaging characteristics, such as hypertensive heart disease and other restrictive cardiomyopathies, for which distinctively different management is required.

In the recent years, scientific interest has focused on the use of nuclear cardiac imaging for the early detection of CA. Planar imaging alone or with single-photon emission computed tomography (SPECT) using nonamyloid-specific, bone-avid radiotracers $\left({ }^{99 \mathrm{~m}} \mathrm{Tc}-\mathrm{DPD}\right.$ [3,3-diphosphono-1,2-propanodicarboxylic acid], ${ }^{99 m}$ Tc-MDP [methylene diphosphonate], ${ }^{99 m}$ Tc-HMDP [hydroxymethylene diphosphonate], and ${ }^{99 m}$ Tc-PYP [pyrophosphate]) have been found to be more effective in detecting TTR myocardial deposits. ${ }^{8-17}$ Recent data 
indicate that the increased level of microcalcifications may explain the enhanced affinity of bone-seeking tracers for TTR over AL amyloidosis. ${ }^{18}$ Amyloidspecific, positron emitting ${ }^{18} \mathrm{~F}$ - and ${ }^{11} \mathrm{C}$-tagged tracers have been used successfully with positron emission tomography (PET) imaging for the identification of both AL and TTR subtypes of CA. ${ }^{19,20}$ However, there still remains some overlap in the diagnoses of AL and TTR$\mathrm{CA}$ and the best strategy for approaching patients with suspected CA is yet to be defined.

In this issue of the Journal of Nuclear Cardiology, Capelli et al retrospectively assessed the diagnostic value of $99 \mathrm{mTc}-\mathrm{HMDP}$ in addition to thorough workup (echocardiography, biomarkers, ECG, cardiac MRI) in a relatively small cohort $(\mathrm{N}=65)$ of patients with biopsy proven CA $(N=26$ AL CA; $N=39$ TTR CA). ${ }^{21}$ Importantly, all patients underwent planar ${ }^{99 \mathrm{~m}} \mathrm{Tc}$ HMDP total body scintigraphy and not SPECT imaging. All patients with TTR-CA had cardiac uptake of TcHMDP based on visual/qualitative evaluation using the Perugini score $(N=3$ grade 1 -mild cardiac uptake, inferior to bone uptake, $N=16$ grade 2-moderate cardiac uptake associated with attenuated bone uptake and $N=20$ grade 3-high cardiac uptake with decreased or absent bone uptake). Quantitative assessment using the heart retention-to-whole body retention ratio (HR:WBR) showed that patients with TTR-CA had a significantly higher HR:WBR value compared to ALCA $(6.2 \pm 1.4$ vs $2.2 \pm .4, P=.0001)$ and also when compared to patients with left ventricular hypertrophy $(\mathrm{LVH})$ not related to $\mathrm{CA}(6.2 \pm 1.4$ vs $1.6 \pm .7$, $P=.0001)$. HR:WBR between AL-CA and LVH patients did not differ significantly, but more importantly did not overlap with the respective value for TTRCA patients. The authors concluded that ${ }^{99 \mathrm{~m}} \mathrm{Tc}$-HMDP total body scintigraphy is an accurate tool to diagnose TTR-CA with a Perugini score of $\geq 2$ carrying $100 \%$ specificity and $93 \%$ sensitivity and a cut-off of 3.3 for HR:WBR carrying a $100 \%$ accuracy for TTR-CA, based on ROC curve analysis. Therefore, ${ }^{99 \mathrm{~m}} \mathrm{Tc}$-HMDP could be considered an effective radiotracer to correctly identify patients with TTR-CA and differentiating them from AL-CA and LVH.

The use of bisphosphonates with planar or SPECT bone scintigraphy in diagnosis of TTR-CA is not a novel concept. Since the seminal work by Perugini et $\mathrm{al}^{8}$, a number of other studies have showed that ${ }^{99 \mathrm{~m}} \mathrm{Tc}-\mathrm{DPD}$ and ${ }^{99 \mathrm{~m}} \mathrm{Tc}-\mathrm{PYP}$ nuclear imaging is feasible and effective in detecting TTR-CA. ${ }^{9,11,12,15-17}$ In addition to the traditionally used qualitative Perugini score which compares myocardial to bone uptake and the semiquantitative HR:WBR, ${ }^{8}$ Bokhari et al also showed that ${ }^{99 M}$ Tc-PYP imaging with a heart-to-contralateral lung ratio of $>1.5$ detected TTR-CA with a $97 \%$ and $100 \%$ sensitivity and specificity, respectively. ${ }^{16}$ Negative and positive example images of TTR-CA with ${ }^{99 \mathrm{M}} \mathrm{Tc}$-PYP imaging are depicted in Figures 1 and 2. Gillmore et al have conducted the largest multicenter study to date, showing that cardiac uptake (Perugini grades 1, 2 or 3) on planar imaging with bisphosphonates $(N=877$ ${ }^{99 \mathrm{~m}}$ Tc-DPD,$\quad N=199{ }^{99 \mathrm{~m}}$ Tc-PYP, $N=141{ }^{99 \mathrm{~m}}$ TcHMDP) is $>99 \%$ sensitive and $68 \%$ specific for detecting TTR-CA, based on the subgroup of patients who had undergone endomyocardial biopsy. ${ }^{17}$ The low specificity resulted largely from low-grade uptake in patients with cardiac AL or cardiac apolipoprotein A-I amyloidosis. The primary goal of the investigators was to assess the specificity and positive predictive value of a positive nuclear scan, so as to better differentiate TTR from AL-CA, given the significant differences in prognosis and treatment strategies. ${ }^{17}$ Indeed, a combination of grade 2 or 3 Perugini score on nuclear bone imaging without a monoclonal protein identified on serum or urine immunoelectrophoresis and serum free light chain assay could reliably diagnose TTR-CA with $100 \%$ specificity and positive predictive value. ${ }^{17,18}$ This was true among the three different bone tracers as well. ${ }^{17}$ One of the conclusions of this study was that ${ }^{99 \mathrm{~m}} \mathrm{Tc}$ HMDP acts very similar to ${ }^{99 \mathrm{~m}} \mathrm{Tc}-\mathrm{DPD}$ and ${ }^{99 \mathrm{~m}} \mathrm{Tc}-\mathrm{PYP}$, the two most widely used tracers. ${ }^{17}$

Despite the utility of the bone-seeking radiotracers in identifying myocardial involvement in TTR-CA, SPECT imaging falls short in the radioactivity quantification at affected sites and thus cannot be used in assessing disease burden and response to therapy. PET is a nuclear modality that can circumvent this problem and is now slowly starting to emerge as a promising tool in the management of CA. ${ }^{11} \mathrm{C}$-PIB (Pittsburgh imaging compound) is a PET tracer able to detect and quantify $\beta$ amyloid fibrils in patients with Alzheimer's disease and was recently shown to also localize and quantify CA. ${ }^{19}$ However, ${ }^{11} \mathrm{C}$ has a very short half-life of $20.4 \mathrm{~min}$ and needs a cyclotron for its production, precluding widespread clinical use. ${ }^{18} \mathrm{~F}$-florbetabir is a novel PET tracer, approved for imaging $\beta$-amyloid protein aggregates in patients with Alzheimer's disease and was recently shown to be useful in distinguishing AL- and TTR-CA from controls, but not AL from TTR-CA in a small pilot study ( $N=9 \mathrm{CA}, N=5$ controls) ${ }^{20}$ (In the same study, ${ }^{18} \mathrm{~F}$-florbetabir also showed promise in quantifying disease activity. ${ }^{20}$ Although the overall myocardial retention index tended to be higher in AL than in TTR patients, none of the indices tested (retention index, LV myocardial SUV, target to background ratio or LV myocardium to liver SUV ratio) clearly distinguished AL from TTR amyloidosis. The higher median ${ }^{18} \mathrm{~F}$ florbetapir retention index in $\mathrm{AL}$ amyloid subjects over ATTR subjects suggested a greater avidity for the light 
A

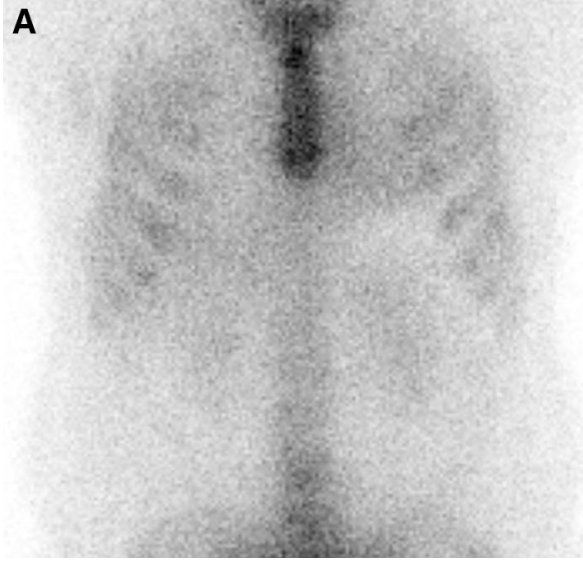

Figure 1. Negative ${ }^{99 \mathrm{~m}}$ Tc-PYP scan (Planar and axial SPECT/CT) with no appreciable myocardial

tracer uptake (Perugini Score Grade 0).

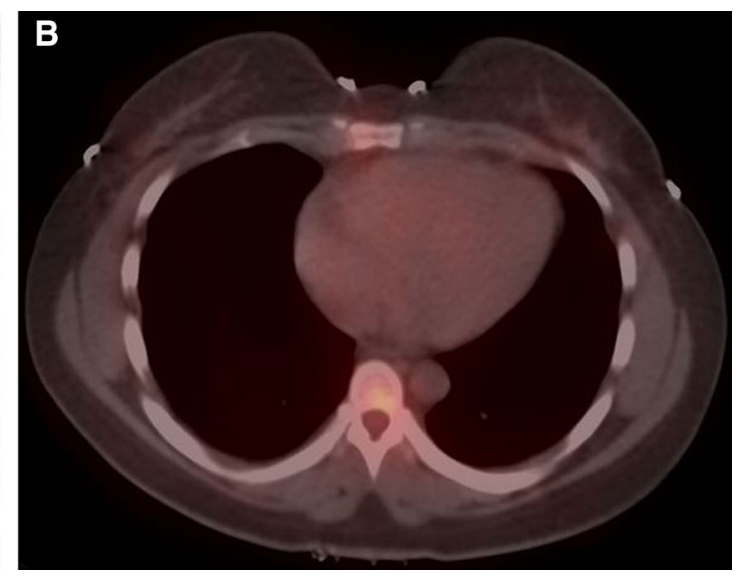

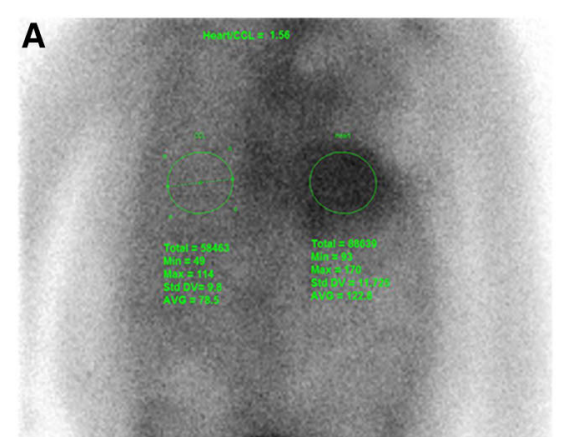
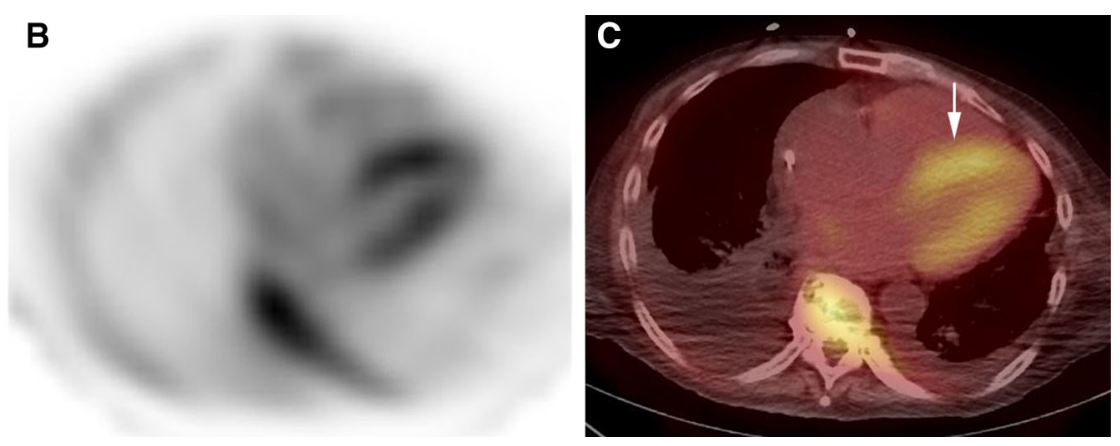

Figure 2. Positive ${ }^{99 \mathrm{~m}}$ Tc-PYP scan (Planar, axial SPECT and axial SPECT/CT) with prominent left ventricular myocardial tracer uptake (arrow) and decreased rib uptake (Perugini Score Grade $3)$. The heart to contralateral ratio is elevated at 1.56 .

chain than for transthyretin protein. The authors suggest an approach of using ${ }^{18} \mathrm{~F}$-florbetabir PET as a screening step for CA (AL or TTR) and if positive, can be followed either by tissue diagnosis or ${ }^{99 \mathrm{~m}} \mathrm{Tc}$ DPD or PYP scanning to confirm TTR myocardial deposits. ${ }^{20}$ Similar findings have been reported with ${ }^{18} \mathrm{~F}$-florbetabir from other laboratories $^{22}$ and with another amyloid specific PET tracer, ${ }^{18} \mathrm{~F}$-florbetaben. ${ }^{23}$

The current study by Capelli et a ${ }^{21}$ confirms earlier findings of smaller studies and case reports ${ }^{13,14}$ as well as the large-scale study by Gillmore et $\mathrm{al}^{17}$ showing the potential of ${ }^{99 \mathrm{~m}} \mathrm{Tc}-\mathrm{HMDP}$ nuclear imaging in patients with suspected TTR-CA, to reliably differentiate those from AL-CA and other causes of LVH. The biggest limitations, as the authors point out, are small sample size and lack of biopsy confirmation in several patients. ${ }^{21}$ Their results are overall positive; however, there are a number of unanswered. Other than local availability, are there specific benefits of using ${ }^{99 \mathrm{~m}} \mathrm{Tc}$ DPD over ${ }^{99 \mathrm{~m}}$ Tc-PYP and ${ }^{99 \mathrm{~m}}$ Tc-HMDP and vice versa, such as ease of labeling (radiochemistry), differences in cost, image quality, radiation exposure etc.? It is also as important (if not more) to define a strategy of non-invasive assessment of patients with suspected $\mathrm{CA}$. We agree with suggestions by Dorbala et $\mathrm{al}^{20}$ for using $18 \mathrm{~F}$ florbetapir PET as a screening tool followed by bone scintigraphy or endomyocardial biopsy in positive cases, to help identify patients with AL CA (positive ${ }^{18} \mathrm{~F}$ florbetabir, negative ${ }^{99 \mathrm{~m}} \mathrm{Tc}-\mathrm{DPD} / \mathrm{PYP} / \mathrm{HMDP}$ ) and TTRCA (positive ${ }^{18} \mathrm{~F}$-florbetabir, positive ${ }^{99 \mathrm{~m}} \mathrm{Tc}-\mathrm{DPD} / \mathrm{PYP} /$ HMDP). In addition to improving our diagnostic capability, more tools are needed to make early diagnosis which can help detect disease prior to its clinical manifestation, to better quantify disease severity, disease burden and response to therapy. ${ }^{18} \mathrm{~F}$-florbetabir appears promising; however, large-scale studies need to be undertaken to definitively establish its value in the management of cardiac amyloidosis. In conclusion, tailored nuclear imaging with PET and SPECT agents may simplify the diagnostic algorithm for cardiac amyloidosis while the PET radiotracer-derived quantitation offers the potential for imaging-guided management. 


\section{Disclosures}

The authors declare that they have no conflict of interest.

\section{References}

1. Kyle RA, Gertz MA. Primary systemic amyloidosis: Clinical and laboratory features in 474 cases. Semin Hematol 1995;32:45-59.

2. Bravo PE, Dorbala $S$. Targeted nuclear imaging probes for cardiac amyloidosis. Curr Cardiol Rep 2017;19:59.

3. Dispenzieri A, Gertz MA, Kyle RA, Lacy MQ, Burritt MF, Therneau TM, et al. Serum cardiac troponins and N-terminal probrain natriuretic peptide: A staging system for primary systemic amyloidosis. J Clin Oncol 2004;22:3751-7.

4. Ng B, Connors LH, Davidoff R, Skinner M, Falk RH. Senile systemic amyloidosis presenting with heart failure: A comparison with light chain-associated amyloidosis. Arch Intern Med 2005; 165:1425-9.

5. Sanchorawala V, Skinner M, Quillen K, Finn KT, Doros G, Seldin DC. Long-term outcome of patients with AL amyloidosis treated with high-dose melphalan and stem-cell transplantation. Blood 2007;110:3561-3.

6. Madan S, Kumar SK, Dispenzieri A, Lacy MQ, Hayman SR, Buadi FK, et al. High-dose melphalan and peripheral blood stem cell transplantation for light-chain amyloidosis with cardiac involvement. Blood 2012;119:1117-22.

7. Ruberg FL, Berk JL. Transthyretin (TTR) cardiac amyloidosis. Circulation 2012;126:1286-300.

8. Perugini E, Guidalotti PL, Salvi F, Cooke RM, Pettinato C, Riva $\mathrm{L}$, et al. Noninvasive etiologic diagnosis of cardiac amyloidosis using 99mTc-3,3-diphosphono-1,2-propanodicarboxylic acid scintigraphy. J Am Coll Cardiol 2005;46:1076-84.

9. Rapezzi C, Quarta CC, Guidalotti PL, Pettinato C, Fanti S, Leone $\mathrm{O}$, et al. Role of $(99 \mathrm{~m}) \mathrm{Tc}-\mathrm{DPD}$ scintigraphy in diagnosis and prognosis of hereditary transthyretin-related cardiac amyloidosis. JACC Cardiovasc Imaging 2011;4:659-70.

10. Rapezzi C, Quarta CC, Guidalotti PL, Longhi S, Pettinato C, Leone $\mathrm{O}$, et al. Usefulness and limitations of $99 \mathrm{mTc}-3,3$-diphosphono-1,2-propanodicarboxylic acid scintigraphy in the aetiological diagnosis of amyloidotic cardiomyopathy. Eur J Nucl Med Mol Imaging 2011;38:470-8.

11. Kristen AV, Scherer K, Buss S, aus dem Siepen F, Haufe S, Bauer $R$, et al. Noninvasive risk stratification of patients with transthyretin amyloidosis. JACC Cardiovasc Imaging 2014;7:502-10.

12. Hutt DF, Quigley AM, Page J, Hall ML, Burniston M, Gopaul D, et al. Utility and limitations of 3,3-diphosphono-1,2-propanodicarboxylic acid scintigraphy in systemic amyloidosis. Eur Heart J Cardiovasc Imaging 2014;15:1289-98.
13. Glaudemans AW, van Rheenen RW, van den Berg MP, Noordzij $\mathrm{W}$, Koole M, Blokzijl $\mathrm{H}$, et al. Bone scintigraphy with (99m)technetium-hydroxymethylene diphosphonate allows early diagnosis of cardiac involvement in patients with transthyretinderived systemic amyloidosis. Amyloid 2014;21:35-44.

14. Galat A, Rosso J, Guellich A, van der Gucht A, Rappeneau S, Bodez D, et al. Usefulness of (99m)Tc-HMDP scintigraphy for the etiologic diagnosis and prognosis of cardiac amyloidosis. Amyloid 2015;22:210-20.

15. Castano A, Haq M, Narotsky DL, Goldsmith J, Weinberg RL, Morgenstern R, et al. Multicenter study of planar technetium 99m pyrophosphate cardiac imaging: Predicting survival for patients with ATTR cardiac amyloidosis. JAMA Cardiol 2016;1(8):880-9.

16. Bokhari S, Castano A, Pozniakoff T, Deslisle S, Latif F, Maurer MS. (99m)Tc-pyrophosphate scintigraphy for differentiating lightchain cardiac amyloidosis from the transthyretin-related familial and senile cardiac amyloidoses. Circ Cardiovasc Imaging 2013;6:195-201.

17. Gillmore JD, Maurer MS, Falk RH, Merlini G, Damy T, Dispenzieri A, et al. Nonbiopsy diagnosis of cardiac transthyretin amyloidosis. Circulation 2016;133:2404-12.

18. Stats MA, Stone JR. Varying levels of small microcalcifications and macrophages in ATTR and AL cardiac amyloidosis: Implications for utilizing nuclear medicine studies to subtype amyloidosis. Cardiovasc Pathol 2016;25:413-7.

19. Antoni G, Lubberink M, Estrada S, Axelsson J, Carlson K, Lindsjö $\mathrm{L}$, et al. In vivo visualization of amyloid deposits in the heart with 11C-PIB and PET. J Nuclear Med 2013;54:213-20.

20. Dorbala S, Vangala D, Semer J, Strader C, Bruyere JR, Di Carli MF, et al. Imaging cardiac amyloidosis: A pilot study using (1)(8)F-florbetapir positron emission tomography. Eur J Nucl Med Mol Imaging 2014;41:1652-62.

21. Cappelli F, Gallini C, Di Mario C, Costanzo EN, Vaggelli L, Tutino

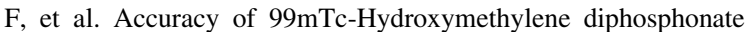
scintigraphy for diagnosis of transthyretin cardiac amyloidosis. J Nucl Cardiol 2017. doi:10.1007/s12350-017-0922-z.

22. Osborne DR, Acuff SN, Stuckey A, Wall JS. A Routine PET/CT Protocol with streamlined calculations for assessing cardiac amyloidosis Using (18)F-florbetapir. Front Cardiovasc Med 2015;2:23.

23. Law WP, Wang WY, Moore PT, Mollee PN, Ng AC. Cardiac amyloid imaging with $18 \mathrm{~F}$-florbetaben PET: A pilot study. J Nucl Med 2016;57:1733-9. 